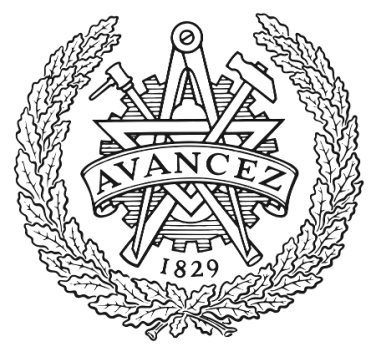

CHALMERS

UNIVERSITY OF TECHNOLOGY

\title{
Augmented Reality with Industrial Process Tomography: To Support Complex Data Analysis in 3D Space
}

Downloaded from: https://research.chalmers.se, 2023-04-26 07:14 UTC

Citation for the original published paper (version of record):

Nowak, A., Zhang, Y., Romanowski, A. et al (2021). Augmented Reality with Industrial Process Tomography: To Support Complex Data Analysis in 3D

Space. UbiComp/ISWC 2021 - Adjunct Proceedings of the 2021 ACM International Joint Conference on Pervasive and Ubiquitous Computing and Proceedings of the 2021 ACM International Symposium on Wearable Computers: 56-58. http://dx.doi.org/10.1145/3460418.3479288

N.B. When citing this work, cite the original published paper. 


\section{Augmented Reality with Industrial Process Tomography: To Support Complex Data Analysis in 3D Space}

\author{
Adam Nowak ${ }^{*}$ \\ Yuchong Zhang* \\ 203151@edu.p.lodz.pl \\ yuchong@chalmers.se \\ Lodz University of Technology \\ Poland \\ Chalmers University of Technology \\ Sweden
}

\author{
Andrzej Romanowski \\ androm@kis.p.lodz.pl \\ Lodz University of Technology \\ Lodz, Poland
}

\author{
Morten Fjeld \\ fjeld@chalmers.se \\ Chalmers University of Technology \\ Gothenburg, Sweden
}

\begin{abstract}
Today, in-situ analyzing and monitoring are imperative for ensuring successful and healthy industrial processes in confined environments. With the rapid development of digitization, augmented reality (AR) has been utilized for letting people immersively interact with the necessary information. However, there are still knowledge gaps between AR technique and domain users pertaining to effective analysis of complex data. Hence, new solutions empowering domain users would benefit the whole industry. In this study, we report an initial prototype supporting complex data visualization and analysis in entire 3D surroundings within industrial process tomography (IPT). Microsoft HoloLens 2 is equipped for users to interact with the 3D information characterizing the workflow of the industrial process with high immersion. Our work distinctly improves the performance compared to existing solutions, pointing the way towards how AR should be deployed and developed more efficiently for aiding IPT systems.
\end{abstract}

\section{CCS CONCEPTS}

- Human-centered computing $\rightarrow$ Empirical studies in collaborative and social computing; Human computer interaction (HCI); Mixed / augmented reality; Visualization systems and tools.

\section{KEYWORDS}

mixed reality, immersion, 3D surroundings, data visualization, industrial process tomography

\section{ACM Reference Format:}

Adam Nowak, Yuchong Zhang, Andrzej Romanowski, and Morten Fjeld. 2021. Augmented Reality with Industrial Process Tomography: To Support Complex Data Analysis in 3D Space. In Adjunct Proceedings of the 2021 ACM International foint Conference on Pervasive and Ubiquitous Computing and Proceedings of the 2021 ACM International Symposium on Wearable Computers (UbiComp-ISWC '21 Adjunct), September 21-26, 2021, Virtual, USA. ACM, New York, NY, USA, 3 pages. https://doi.org/10.1145/3460418.3479288

${ }^{*}$ Both authors contributed equally to this research.

Permission to make digital or hard copies of part or all of this work for personal or classroom use is granted without fee provided that copies are not made or distributed for profit or commercial advantage and that copies bear this notice and the full citation on the first page. Copyrights for third-party components of this work must be honored For all other uses, contact the owner/author(s).

UbiComp-ISWC '21 Adjunct, September 21-26, 2021, Virtual, USA

(c) 2021 Copyright held by the owner/author(s).

ACM ISBN 978-1-4503-8461-2/21/09.

https://doi.org/10.1145/3460418.3479288

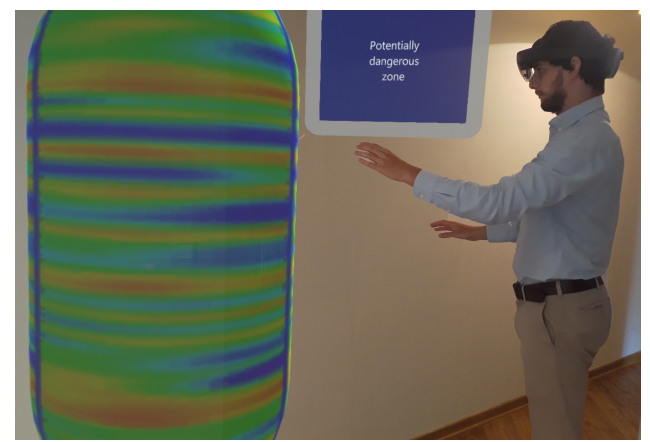

Figure 1: User interacts with 3D complex data presentation.

\section{INTRODUCTION AND RELATED WORK}

Augmented Reality (AR), a derivative from Virtual Reality (VR) technique whereby the virtual objects are overlaid in the real environment [1], is one of the cutting-edge technologies being applied in various fields such as medicine and education [2]. For industry, there is still a gap between AR technique and industrial application, resulting in AR not being more extensively-used [4]. However, on the premise that it has the capacity to offer the interactive interfaces of displayed digital content, while bringing users to immersively communicate and interact with the virtual objects, AR has already been deployed in some practical industrial aspects $[3,5,7]$.

Industrial process tomography (IPT), as a widely used non-intrusion imaging technique, has effectively demonstrated its high value in industrial process monitoring and product quality control [11, 12]. Electrical Capacitance Tomography (ECT) is a specific mechanism to monitor processes in confined containers, with no access to the internal content of the targeted vessel [10]. Complex data analysis always requires efficient reproduction of the measurement regarding the reconstructed images by ECT to reveal the material distribution inside the closed containers [6]. It is critical to observe and analyze the images which imply the state of the process flow, supported with tomograms and graphs, in a protection-friendly manner without any direct interference with the on-going industrial process [9]. Therefore, getting domain users instant access for implementing in-situ data observation and analysis becomes the main concern of this paper. Hereby, we propose a novel AR solution for IPT users to interact with complex data by eliciting the immersive and tangible 3D surroundings. 
Although exploiting Mixed Reality (MR) in IPT systems is still scarce, some research has already pioneered the usage of AR to benefit the data analysis for industrial tomographic users [9, 13]. At the beginning of this century, Mann et al. [8] brought an AR methodology so as to visualize the mixed fluid in stirred chemical reactors by using ECT. Recently, Nowak et. al [9] demonstrated a proof-of-concept study regarding ECT, by developing a new AR solution to underpin the onsite analysis of complex numerical data through mutual collaboration. In our study, we propose a updated solution based on Nowak's [9], to help users investigate more complicated data which are represented as images or graphs in a more immersive and interactive 3D environment.

\section{PROPOSED SOLUTION}

\subsection{System Features}

The proposed system is manifested as an interactive application in line with AR/MR technique to support domain users for in-situ ECT data analysis. The practical application is capable of displaying a variety of visualized data especially in $3 \mathrm{D}$ format, characterizing the workflow of the carried industrial process. During implementation, users are requested to be equipped with the up-to-date Microsoft HoloLens 2 (the link), a convenient and portable headset which provides the most comfortable and immersive experience for AR/MR.

\subsection{Initial application}

The main goal of our prototype is to provide users with an entirely immersive experience to interact with the ECT complex data in a 3D surrounding. By using Microsoft HoloLens 2, users are enabled to observe, interact, and analyze different 2D and 3D graphical information from the original source. An example of data in a raw format browsed through the headset is displayed in Figure 1. Then, the combined formats of the complex data such as graphs or plots, followed by the ultimate $3 \mathrm{D}$ reconstructions of the process are presented to the users. All the virtual objects are shown as holographic artifacts floating around, which makes users able to manipulate, move, scale, and rotate them with hands in this completely immersive environment, as elaborated in Figure 2.
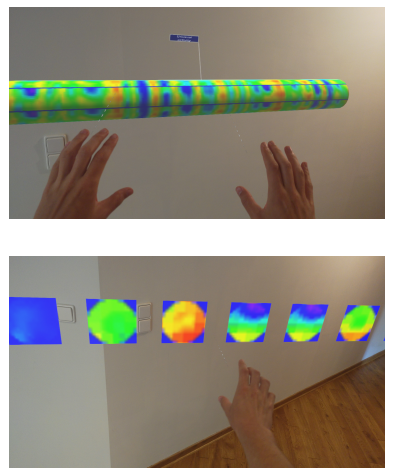

Figure 2: User works with different forms of data visualisation. Left: 3D tomogram data visualisation, Right: raw data frames representing different flows over time.

\section{DISCUSSION}

Novel tools and applications are desirable for industrial ecosystems. New cutting edge technologies will enhance humans' performance within industrial domain. We strongly believe that shifting the working environment from flat 2D screens to stereoscopic 3D space can immerse users more deeply into the context which enhances the working efficiency. However, we acknowledge that there are still some limitations in our solution. First, we have to realize current hardware limitations, such as available computing power, battery capacity, device weight, and field of view will have observable impact on potential applications. Also, it may initially be difficult for users to adjust to our new AR system, where they are requested to become familiarized with all the application details. Last but not least, we have not yet tested our solution regarding rigorous human aspects, which is obligatory for a human-in-the-loop technique.

\section{CONCLUSIONS AND FUTURE WORK}

In this study, we present an early-stage prototype enhancing immersion for complex data analysis for IPT experts using AR technology in an entire 3D space. Our novel solution uses the newest AR/MR headset, Microsoft HoloLens 2, which facilitates displaying different formats of data - from raw ECT data frames, through processed graphs and plots, and finally complex 3D representation related to specific IPT processes. Currently our app is under further development and is not ready to be tested in real life scenarios. For future work, we will identify the following features: voice commands to control virtual environment, shared experience allowing users for mutual collaboration on the spot, and connecting data streams from sensors to have in-situ preview, which will all be implemented with a more comprehensive experiment with distinguished IPT experts.

\section{REFERENCES}

[1] Ronald T Azuma. 1997. A survey of augmented reality. Presence: Teleoperators \& Virtual Environments 6, 4 (1997), 355-385.

[2] Mark Billinghurst, Adrian Clark, Gun Lee, et al. 2015. A Survey of Augmented Reality. Foundations and Trends ${ }^{\circledR}$ in Human-Computer Interaction 8, 2-3 (2015), 73-272.

[3] Răzvan Gabriel Boboc, Florin Gîrbacia, and Eugen Valentin Butilă. 2020. The application of augmented reality in the automotive industry: A systematic literature review. Applied Sciences 10, 12 (2020), 4259.

[4] Luís Fernando de Souza Cardoso, Flávia Cristina Martins Queiroz Mariano, and Ezequiel Roberto Zorzal. 2020. A survey of industrial augmented reality. Computers \& Industrial Engineering 139 (2020), 106159

[5] Paula Fraga-Lamas, Tiago M Fernández-Caramés, Óscar Blanco-Novoa, and Miguel A Vilar-Montesinos. 2018. A review on industrial augmented reality systems for the industry 4.0 shipyard. Ieee Access 6 (2018), 13358-13375.

[6] Krzysztof Grudzien. 2017. Visualization system for large-scale silo flow monitoring based on ECT technique. IEEE Sensors fournal 17, 24 (2017), 8242-8250.

[7] Idris Ismail, JC Gamio, SF Ahmed Bukhari, and WQ Yang. 2005. Tomography for multi-phase flow measurement in the oil industry. Flow measurement and instrumentation 16, 2-3 (2005), 145-155.

[8] Reg Mann, SJ Stanley, Dimiter Vlaev, Eustace Wabo, and Ken Primrose. 2001. Augmented-reality visualization of fluid mixing in stirred chemical reactors using electrical resistance tomography. Fournal of Electronic Imaging 10, 3 (2001), 620-629.

[9] Adam Nowak, Mikołaj Woźniak, Zdzisława Rowińska, Krzysztof Grudzień, and Andrzej Romanowski. 2019. Towards in-situ process tomography data processing using augmented reality technology. In Adjunct Proceedings of the 2019 ACM International foint Conference on Pervasive and Ubiquitous Computing and Proceedings of the 2019 ACM International Symposium on Wearable Computers. 168-171.

[10] Andrzej Romanowski, Krzysztof Grudzien, Zbigniew Chaniecki, and Pawel Wozniak. 2013. Contextual processing of ECT measurement information towards detection of process emergency states. In 13th International Conference on Hybrid Intelligent Systems (HIS 2013). IEEE, 291-297. 
[11] Yuchong Zhang and Morten Fjeld. 2020. Condition monitoring for confined industrial process based on infrared images by using deep neural network and variants. In Proceedings of the 2020 2nd International Conference on Image, Video and Signal Processing. 99-106.

[12] Yuchong Zhang, Morten Fjeld, Marco Fratarcangeli, Alan Said, and Shengdong Zhao. 2021. Affective Colormap Design for Accurate Visual Comprehension in
Industrial Tomography. Sensors 21, 14 (2021), 4766.

[13] Yuchong Zhang, Rahul Yadav, Adel Omrani, and Morten Fjeld. 2021. A Novel Augmented Reality System To Support Volumetric Visualization in Industrial Process Tomography. In Proceedings of the 2021 Conference on Interfaces and Human Computer Interaction. 3-9. 